\title{
Tratamiento de los derrames pleurales malignos con quimioterapia sistémica, después del drenaje con tubo de tórax
}

\author{
Tratamiento derrames pleurales con quimioterapia \\ Pleural effusions treatment with chemotherapy

\begin{abstract}
- Héctor González1, Hugo Londoño², Yina Mancera³
Sección de Oncología Clínica, Clínica Manuel Elkin Patarroyo. Instituto de Seguros Sociales (Ibagué, Colombia).

Sección de Cirugía, Clínica Manuel Elkin Patarroyo. Instituto de Seguros Sociales (Ibagué, Colombia).
\end{abstract} \\ Sección de Estadística, Zema (Bogotá, D.C., Colombia).
}

\section{Resumen}

Introducción: los derrames pleurales son una complicación frecuente en el cáncer. Son generalmente causados por compromiso pleural tumoral. El diagnóstico es clínico y paraclínico. Las modalidades terapéuticas incluyen la pleurodesis química, que no es etiológica ni fisiológica. La quimioterapia en cánceres quimiosensibles es una buena alternativa.

El objetivo de este estudio fue describir la frecuencia de respuesta a la quimioterapia sistémica en derrames pleurales después del drenaje.

Materiales y métodos: se trata de un estudio descriptivo tipo serie de casos. Se incluyeron pacientes con cánceres quimiosensibles, derrame pleural maligno, sin quimioterapia previa, IK $>80$, expectativa de vida $>6$ meses. Procedimiento: práctica de Rx tórax, inserción del tubo para drenaje evacuante, aplicación de quimioterapia sistémica, remoción del tubo al día siguiente de terminar la quimioterapia. No reproducción del derrame mínimo por seis meses. Se utilizó análisis de estadística descriptiva.

Resultados: se estudiaron 69 pacientes: hombres (37,68\%), mujeres (62,31\%). Edad promedio: 59 años. Cánceres: carcinoma broncogénico $(43,47 \%)$, carcinoma de mama $(28,98 \%)$, carcinoma de ovario $(14,49 \%)$, carcinoma de origen desconocido $(7,24 \%)$, otros (5,79\%). Estadios clínicos: IIIb (57,97\%), IV (42,02\%). Respuesta total: 85,5\%. Se encontró respuestas en cánceres de origen conocido del $90,6 \%$ y en cánceres de origen desconocido del $20 \%$. Recidiva del derrame pleural en un $14,5 \%$.

Conclusiones: la quimioterapia sistémica después de la evacuación del derrame es una buena alternativa para tratar derrames pleurales malignos quimiosensibles, porque es etiológica y tiene una alta tasa de respuesta.

Palabras clave: derrame pleural maligno, tratamiento farmacológico, quimioterapia sistémica, tubo de tórax.

\begin{abstract}
Introduction: Pleural effusions are a common complication in cancer. They are usually caused by tumor pleural involvement. The diagnosis is clinical and paraclinical. The therapeutic modalities include chemical pleurodesis, which is not etiological or physiological. Chemotherapy in chemosensitive cancers is a good alternative. The objective of this study was to describe the frecuency of responses to systemic chemotherapy in pleural effusions after drainage.

Materials and methods: We included patients with chemosensitive cancers, malignant pleural effusion, no previous chemotherapy, $\mathrm{KI}>80$, life expectancy $>6$ months. Procedure: Rx thorax, insertion of the tube for evacuating drainage, systemic chemotherapy application, tube removal the day after finishing the chemotherapy. No reproduction of the minimum spill for 6 months. A descriptive statistical analysis was used.

Results: 69 patients were studied: Men (37.68\%), Women (62.31\%). Average age: 59 years. Cancers: bronchogenic carcinoma $(43.47 \%)$, breast carcinoma $(28.98 \%)$, ovarian carcinoma $(14.49 \%)$, carcinoma of unknown origin $(7.24 \%)$, others (5.79\%). Clinical states: IIIb (57.97\%), IV (42.02\%). Total response: $85.5 \%$. An response was found in cancers of known origin of $90.6 \%$ and in cancers of unknown origin of $20 \%$. Recurrence of pleural effusion $14.5 \%$.

Conclusions: Systemic chemotherapy after evacuation of the effusion is a good alternative to treat malignant chemosensitive pleural effusions, because it is etiological and has a high response.
\end{abstract}

Key words: Pleural effusion, malignant, drug therapy. 


\section{Introducción}

Los derrames pleurales malignos (DPM) son el resultado de la diseminación metastásica al espacio pleural de una variedad de tumores primarios, con frecuencia pulmonares, mamarios, gastrointestinales, ginecológicos y hematológicos' ${ }^{1}$ los cuales comprenden cerca del $50 \%$ de las causas de derrame pleural en los pacientes adultos $^{2-6}$. Los carcinomas pulmonares y mamarios son los tumores metastásicos más comunes en la pleura en hombres y mujeres, respectivamente ${ }^{7}$. Todos los tumores, con excepción de los del SNC, pueden producir derrames pleurales ${ }^{2,3}$.

Los principales mecanismos patogénicos incluyen metástasis pleurales por aumento de la permeabilidad y disminución de la reabsorción, compromiso tumoral de ganglios mediastinales, obstrucción bronquial por reducción de presiones, neumonía, compromiso del conducto torácico, hipoproteinemia, tromboembolismo pulmonar, posradioterapia ${ }^{6,8-12}$. Los tumores pueden alterar el delicado balance de formación y reabsorción del fluido pleural, conduciendo a su acumulación por diferentes mecanismos ${ }^{8-12}$.

Los derrames pleurales malignos son exudados. La mayoría de derrames pleurales que son exudados, en ausencia de neumonía, son malignos, sin importar el resultado de la citología ${ }^{13-16}$. La biopsia pleural es positiva en el 50\%-85\% de los casos, convirtiéndose en un elemento fundamental en el diagnóstico, cobrando importancia para la realización de estudios de inmunohistoquímica en el caso de carcinomas metastásicos de origen desconocido ${ }^{17}$. El diagnóstico es clínico y paraclínico, siendo los principales síntomas tos y disnea, y los signos disminución o abolición del murmullo vesicular y matidez a la percusión. El principal objetivo del tratamiento del derrame pleural es la mejoría de los síntomas.

Entre las diferentes modalidades terapéuticas para el derrame pleural, están la toracentesis, toracostomía con drenaje cerrado, pleurectomía, derivaciones pleuroperitoneales y la pleurodesis química, la cual es bastante utilizada, pero no es etiológica ni fisiológica ${ }^{18,19}$; además, se han reportado en diferentes estudios los efectos de la absorción sistémica de algunos de los agentes empleados en dicho procedimiento, que pueden estar relacionados con síndrome de dificultad respiratoria aguda en algunas ocasiones ${ }^{20,21}$
Los catéteres pleurales permanentes son una alternativa cada vez más usada para reemplazar la pleurodesis, permitiendo el drenaje domiciliario y manejo ambulatorio, aunque se asocia más con efectos adversos ${ }^{22}$.

La quimioterapia sistémica en cánceres quimiosensibles es una buena alternativa, de manejo esencialmente paliativo. En particular, para las metástasis pulmonares, esta quimioterapia es un factor muy importante en su tratamiento multimodal en otros tipos histológicos, como el osteosarcoma, cáncer de mama, tumores de células germinales y coriocarcinoma. La elección y la secuencia de tratamientos se encuentran influenciadas por la histología del tumor, la presencia o ausencia de mutaciones y los factores individuales de cada paciente, como el estado general, edad, comorbilidades y preferencias con respecto al tratamiento ${ }^{23}$.

El objetivo de este estudio fue describir la frecuencia de respuestas a quimioterapia sistémica en el manejo de los derrames pleurales malignos después de su drenaje o evacuación.

\section{Materiales y métodos}

Estudio descriptivo, donde se realizó de forma prospectiva seguimiento a pacientes con diagnóstico de cáncer que son quimiosensibles y que presentaron derrame pleural maligno en el período comprendido entre el $1^{\circ}$ de marzo de 2001 y el 30 de julio de 2009, en la Sección de Oncología de la Clínica Manuel Elkin Patarroyo en Ibagué (Colombia).

Se establecieron como criterios de inclusión para el estudio tener una edad mayor a 14 años, presentar derrame pleural maligno, no tener tratamiento con quimioterapia previa, poseer una perspectiva de vida mayor a seis meses y reportar un índice funcional Karnofsky mayor de 80. Se excluyeron los pacientes con derrame pleural recidivante a este manejo. El procedimiento del tratamiento del derrame pleural maligno se expone en la figura 1.

Para analizar la evolución de los pacientes respecto de la quimioterapia, se evaluó la presencia de reproducción tumoral a seis meses a través de aspectos clínicos e imaginológicos. Para el análisis de los resultados, se utilizó estadística descriptiva mediante medidas de tendencia central y frecuencias absolutas de los desenlaces evaluados. 


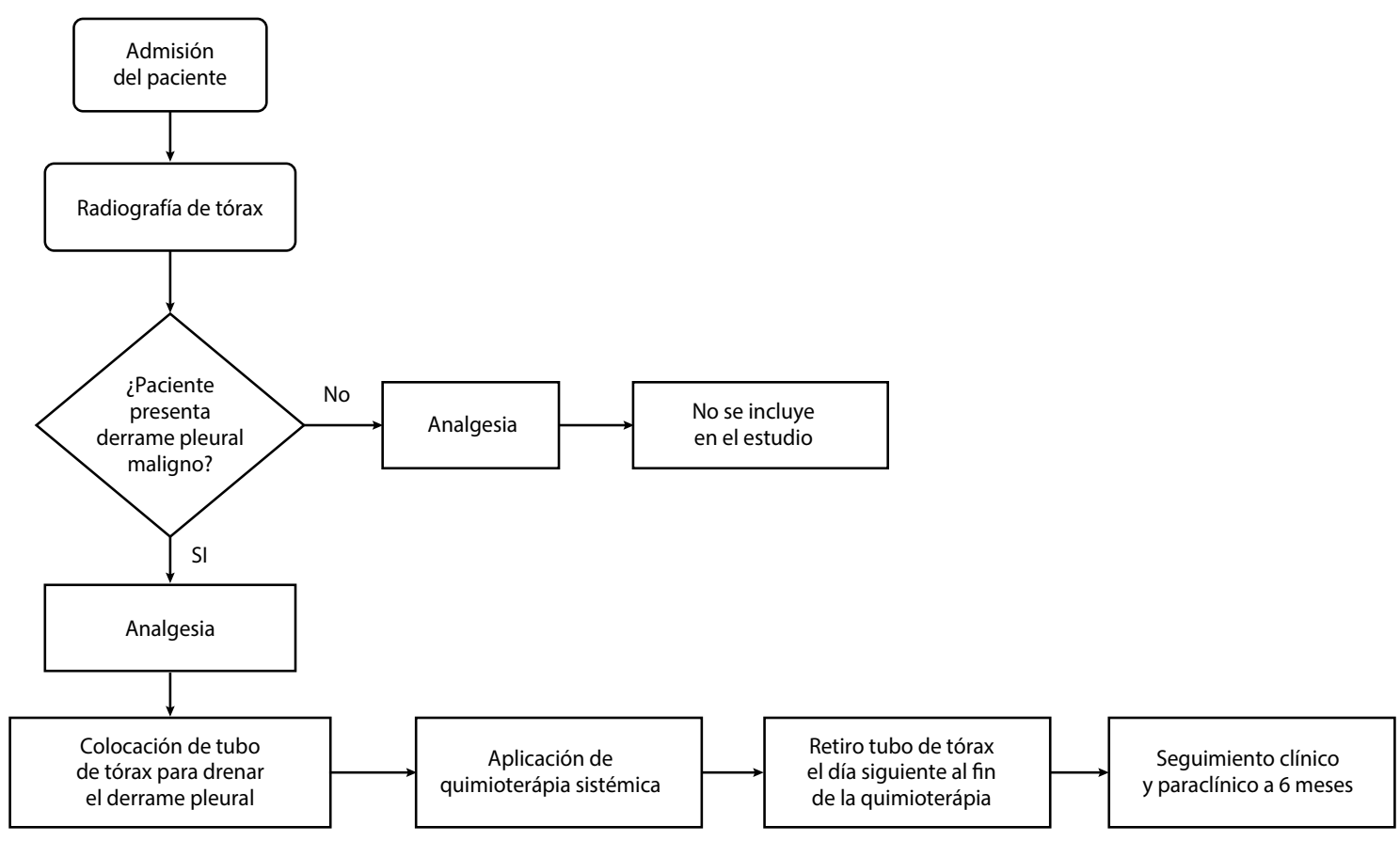

Figura 1. Procedimiento derrame pleural maligno.

\section{Resultados}

En total, fueron incluidos 69 pacientes en el estudio, de los cuales 26 (37,68\%) eran de sexo masculino y 43 (62,31\%) femenino. La edad promedio de los pacientes fue de 57 años, con una mediana de 59 años.

Las causas de derrame pleural se muestran en la tabla 1. En los hombres, la causa más común de derrame fue el carcinoma broncogénico (84,61\%) con 22 pacientes y en mujeres el carcinoma de seno $(46,5 \%)$ con 20 pacientes.

Tabla 1. Causas de derrame pleural

\begin{tabular}{|l|c|c|}
\hline \multicolumn{1}{|c|}{ Causa } & n & $\%$ \\
\hline Carcinoma broncogénico & 30 & 43,47 \\
\hline Carcinoma de seno & 20 & 28,98 \\
\hline Carcinoma de ovario & 10 & 14,49 \\
\hline Carcinoma metastásico de origen desconocido & 5 & 7,24 \\
\hline Otros: vías biliares, melanoma maligno & 4 & 5,79 \\
\hline Total & $\mathbf{6 9}$ & $\mathbf{1 0 0}$ \\
\hline
\end{tabular}

Los estadios clínicos presentes en los pacientes del estudio fueron el IIIb, en 40 pacientes $(57,97 \%$ ) y el IV en 29 pacientes (42,02\%). Los medicamentos empleados en quimioterapia para los pacientes fueron carboplatino, etopósido, ciclofosfamida, cisplatino, docetaxel, paclitaxel, gemcitabina, doxorrubicina. En la tabla 2 se muestran los esquemas empleados en el tratamiento de los pacientes.

Tabla 2. Esquemas de quimioterapia empleados

\begin{tabular}{|l|c|c|}
\hline \multicolumn{1}{|c|}{ Esquema } & n & $\%$ \\
\hline Carbopatino-etopósido & 7 & 10,1 \\
\hline Cisplatino-etopósido & 10 & 14,5 \\
\hline Cisplatino-gemcitabina & 3 & 4,4 \\
\hline Ciclofosfamida-carboplatino & 8 & 11,6 \\
\hline Gemcitabina-vinorelbine & 2 & 2,9 \\
\hline Docetaxel-gemcitabina & 3 & 4,3 \\
\hline Paclitaxel-gemcitabina & 2 & 2,9 \\
\hline Docetaxel o paclitaxel-cisplatino o carboplatino & 10 & 14,5 \\
\hline Doxorrubicina-ciclofosfamida & 20 & 29,0 \\
\hline Capecitabina (docetaxel o gemcitabina) & 4 & 5,8 \\
\hline Total & $\mathbf{6 9}$ & $\mathbf{1 0 0}$ \\
\hline
\end{tabular}

En cuanto a los resultados del tratamiento con quimioterapia, se evidenció una similitud en las principales causas tumorales (broncogénico, seno y ovario). La frecuencia de respuesta en cánceres de origen conocido fue resolución en el 90,6\%, mientras que en carcinomas metastásicos de origen desconocido fue del $20 \%$. Diez de los pacientes presentaron reproducción tumoral $(14,5 \%)$ para el mismo período de tiempo posterior al tratamiento (tabla 3). 
Tabla 3. Estado del derrame pleural maligno a seis meses

\begin{tabular}{|l|c|c|}
\hline \multicolumn{1}{|c|}{ Estado del DPM } & N & \% \\
\hline No reproducción derrame pleural & 59 & 85,5 \\
\hline Reproducción derrame pleural & 10 & 14,5 \\
\hline Total & $\mathbf{6 9}$ & $\mathbf{1 0 0}$ \\
\hline
\end{tabular}

\section{Discusión}

La quimioterapia sistémica después del drenaje con tubo de tórax, mediante toracostomía con drenaje cerrado, es una buena alternativa para el tratamiento de los derrames pleurales malignos en cánceres quimiosensibles, porque es etiológica y tiene una alta respuesta ${ }^{24}$. Reforzando lo reportado en la literatura, en este estudio no se presentó reproducción tumoral en el $85,5 \%$ de los casos a seis meses.

La desaparición del derrame pleural se considera que es secundaria a la acción de las drogas citotóxicas sobre las células malignas que se encuentran en la pleura y el líquido pleural, siendo este el mecanismo fundamental del resultado ${ }^{11,25}$. Para que este método sea efectivo, es necesario drenar el derrame ${ }^{26}$ y que el cáncer sea quimiosensible. Estos derrames, especialmente los que se desarrollan de manera temprana en el curso de la enfermedad, son generalmente sensibles a la quimioterapia y pueden responder dramáticamente ${ }^{10}$.
Los derrames que se desarrollan tardíamente en el curso de la enfermedad y aquellos que aparecen en pacientes ya tratados con quimioterapia previamente son usualmente resistentes a la quimioterapia. Igualmente, el porcentaje de respuesta a la quimioterapia es menor en carcinomas metastásicos de origen desconocido, ya que no siempre se puede aplicar el esquema de quimioterapia más eficaz para cada tipo de cáncer.

En cuanto a las características de la población del estudio, se presentaron más mujeres que hombres, debido a la gran diferencia en la incidencia del cáncer de mama en las mujeres, comparado con el bajo porcentaje en los hombres. Adicionalmente, el carcinoma broncogénico fue el más común en toda la población de pacientes y entre la población masculina. Los estadios clínicos tardíos fueron los más comunes (III y IV), como corresponde a la presentación de derrames pleurales en estos estadios en los cánceres más representativos dentro del estudio.

Esta modalidad de tratamiento podría mejorar la sobrevida de los pacientes, siendo importante establecerla en pacientes con un alto índice funcional, no obstante ser un tratamiento paliativo. En investigaciones futuras, se podrían discutir otros mecanismos, como la producción de pleurodesis, por la inflamación generada por los antineoplásicos en la superficie pleural.

\section{Referencias}

1. Villena V, Cases E, Fernández A, De Pablo A, Pérez E, Porcel $J M$, et al. Normativa sobre el diagnóstico y tratamiento del derrame pleural. Actualización. Arch Bronconeumol [internet]. 2014;50(6):235-49. Disponible en: http://dx.doi.org/10.1016/j. arbres.2014.01.016

2. Leuallen EC, Carr DT. Pleural efussion: a statistical study of 436 patients. N Engl J Med. 1955;252(3):79-83.

3. Storey $D D$, Dines $D E$, Coles DT. Pleural effusion. A diagnostic dilemma. JAMA. 1976;236(19):2183-6.

4. Hirsch $A$, Ruffie $P$, Nebut $M$, Bignon J, Chrétien J. Pleural effusion: laboratory tests in 300 cases. Thorax. 1979;34(1):106-12.

5. Tinney $W$, Olsen $A$. The significance of fluid in the pleural space: a study of 274 cases. J Thorac Surg. 1945;14:248-52.

6. Friedman MA, Slater E. Malignant pleural effusions. Cancer Treat Rev. 1978;5(2):49-66

7. Light RW, George RB. Incidence and significance of pleural effusion after abdominal surgery. Chest [internet]. 1976;69(5):6215. Disponible en: http://dx.doi.org/10.1378/chest.69.5.621

8. Black LF. The pleural space and pleural fluid. Mayo Clin Proc. 1972;47(7):493-506

9. Austin EH, Flye MW. The treament of recurrent malignant pleural effusion. Ann Thorac Surg. 1979;28(2):190-203.
10. Meyer PC. Metastatic carcinoma of the pleura. Thorax. 1966;21(5):437-43.

11. Chernow $B$, Sahn SA. Carcinomatous involvement of the pleura: an analysis of 96 patients. Am J Med. 1977;63(5):695-702.

12. Devita $V$, Hellman $S$, Rosenberg $S$, editors. Cancer: principles and practice of oncology. $6^{\text {th }}$ ed. Lippincott Williams \& Wilkins; 2001

13. Light RW, Macgregor MI, Luchsinger PC, Ball WC Jr. Pleural effusions: the diagnostic separation of transudates and exudates. Ann Intern Med. 1972;77(4):507-13.

14. Sahn SA. The differential diagnosis of pleural effusions. West J Med. 1982;137(2):99-108.

15. Casciato DA, Territo MC. Manual of clinical oncology. $6^{\text {th }}$ ed. Philadelphia: Lippincott Williams \& Wilkins; 2009.

16. Noppen M. Normal volume and cellular contents of pleural fluid. Curr Opin Pulm Med. 2001;7(4):180-2.

17. Heffner JE. Diagnosis and management of malignant pleural effusions. Respirology. 2008;13(1):5-20.

18. Ruckdeschel JC, Moores D, Lee JY, Einhorn LH, Mandelbaum I, Koeller J, et al. Intrapleural therapy for malignant pleural effusions. A randomized comparison of bleomycin and tetracycline. Chest. 1991;100(6):1528-35. 
19. Rosato FE, Wallach MW, Rosato EF. The management of malignant effusions from breast cancer. J Surg Oncol. 1974;6(5):441-9.

20. Terra RM, Bellato RT, Teixeira LR, Chate RC, Pego-Fernandes PM. Safety and systemic consequences of pleurodesis with three different doses of silver nitrate in patients with malignant pleural effusion. Respiration. 2015;89(4):276-83.

21. Maskell NA, Lee YC, Gleeson FV, Hedley EL, Pengelly G, Davies RJ. Randomized trials describing lung inflammation after pleurodesis with talc of varying particle size. Am J Respir Crit Care Med. 2004;170(4):377-82.

22. Davies $H E$, Mishra EK, Kahan $B C$, Wrightson JM, Stanton $A E$, Guhan A, et al. Effect of an indwelling pleural catheter vs chest tube and talc pleurodesis for relieving dyspnea in patients with malignant pleural effusion: the TIME2 randomized controlled trial. JAMA. 2012;307(22):2383-9.

23. IFC, Herrero Burgos J, Jiménez Orozco E. Manejo del pulmón metastático. En: Manual de diagnóstico y terapéutica en neumología. $3^{a}$ ed. Sevilla, España: Neumosur; 2016. p. 651-8.

24. Hill KM, Muers MF. Palliative care for patients with non-malignant end stage respiratory disease. Thorax. 2000;55(12):979-81.

25. Skeel $R$, Khlief $S$. Handbook of cancer chemotherapy. $8^{\text {th }}$ ed. Lippincott Williams \& Wilkins; 2011.

26. Anderson CB, Philpott GW, Fergusson TB. The treatment of malingnant pleural effusions. Cancer. 1974;33(4):916-22. 